\title{
Modulation of the voltage-dependent anion-selective channel by cytoplasmic proteins from wild type and the channel depleted cells of Saccharomyces cerevisiae
}

\author{
Hanna Kmita ${ }^{\bowtie}$, Małgorzata Budzińska and Olgierd Stobienia \\ Department of Bioenergetics, Institute of Molecular Biology and Biotechnology, \\ Poznan University, Poznań, Poland
}

Received: 10 April, 2003; revised: 15 May, 2003; accepted: 26 May, 2003

Key words: yeast, Saccharomyces cerevisiae, mitochondria, reconstituted system, voltage dependent anion selective channel (VDAC), VDAC-depleted mutant

\begin{abstract}
It is well known that effective exchange of metabolites between mitochondria and the cytoplasm is essential for cell physiology. The key step of the exchange is transport across the mitochondrial outer membrane, which is supported by the voltage-dependent anion-selective channel (VDAC). Therefore, it is clear that the permeability of VDAC must be regulated to adjust its activity to the actual cell needs. VDAC-modulating activities, often referred to as the VDAC modulator, were identified in the intermembrane space of different organism mitochondria but the responsible protein(s) has not been identified as yet. Because the VDAC modulator was reported to act on VDAC of intact mitochondria when added to the cytoplasmic side it has been speculated that a similar modulating activity might be present in the cytoplasm. To check the speculation we used mitochondria of the yeast Saccharomyces cerevisiae as they constitute a perfect model to study VDAC modulation. The mitochondria contain only a single isoform of VDAC and it is possible to obtain viable mutants devoid of the channel ( $\triangle$ por 1$)$. Moreover, we have recently characterised a VDAC-modulating activity located in the intermembrane space of wild type and $\Delta$ por 1 $S$. cerevisiae mitochondria. Here, we report that the cytoplasm of wild type and $\Delta$ por 1 cells of $S$. cerevisiae contains a VDAC-modulating activity as measured in a reconstituted system and with intact mitochondria. Since quantitative differences were observed between the modulating fractions isolated from wild type and $\Delta$ por 1 cells when they were studied with intact wild type mitochondria as well as by protein elec-
\end{abstract}

\footnotetext{
${ }^{\star}$ Part of the results were presented at the 37th Meeting of the Polish Biochemical Society, Wrocław 2002, Poland.

${ }^{凶}$ Corresponding author: Hanna Kmita, Department of Bioenergetics, Institute of Molecular Biology and Biotechnology, Adam Mickiewicz University, A. Fredry 10, 61-701 Poznań, Poland; phone: (48 61) 829 4532, fax: (48 61) 829 4503, e-mail: kmita@main.amu.edu.pl.
}

Abbreviations: BSA, bovine serum albumin; CMF, cytoplasmic modulator fraction displaying VDACmodulating activity; TOM, translocase of the outer membrane; $\mathrm{TPP}^{+}$, tetraphenylophosphonium; VDAC, voltage-dependent anion-selective channel. 
trophoresis it might be concluded that VDAC may influence the properties of the involved cytoplasmic proteins. Moreover, the VDAC-modulating activity in the cytoplasm differs distinctly from that reported for the mitochondrial intermembrane space. Nevertheless, both these activities may contribute efficiently to VDAC regulation. Thus, the identification of the proteins is very important.

The flux of metabolites across the mitochondrial outer membrane is supported by the voltage-dependent anion-selective channel (VDAC), also known as mitochondrial porin (Schein et al., 1976; Colombini, 1979; Benz, 1994; Colombini et al., 1996; Blachly-Dyson \& Forte, 2001). The channel is usually present as isoforms encoded by separated genes. The isoforms display different channel-forming activities and probably play different roles (Blachly-Dyson et al., 1993; 1997; Heins et al., 1994; Elkeles et al., 1997; Sampson et al., 1997; Xu et al., 1999). In the yeast Saccharomyces cerevisiae only a single isoform of VDAC has been identified showing properties that are highly conserved among other species (Blachly-Dyson et al., 1997; Lee et al., 1998). It is formed by the VDAC1 protein (or porin1) encoded by the POR 1 gene. The second VDAC protein present in $S$. cerevisiae mitochondria (called VDAC2 or porin2) is encoded by the POR2 gene and does not display the channel forming activity. Therefore, $S$. cerevisiae can be regarded as a useful model to study VDAC modulation.

It is clear that cellular proteins able to modulate the functional state of VDAC could contribute importantly to the mitochondrial outer membrane permeability. Known regulators of VDAC are the pro- and anti-apoptotic BCL-2 family members but the role of these proteins in VDAC modulation is still not clear (Shimizu et al., 2000; Vander Heiden et al., 1999; 2000; Tsujimoto \& Shimizu, 2002; Crompton et al., 2002). VDAC-modulating activities have also been shown to exist in the intermembrane space of mitochondria of different organisms (Elkeles et al., 1997; Holden \& Colombini, 1988; Liu \& Colombini, 1992; Holden \& Colombini, 1993; Liu et al., 1994; Stobienia et al., 2002). These activities, often referred to as the VDAC modulator, were studied mainly in reconstituted systems (Elkeles et al., 1997; Holden \& Colombini, 1988; Holden \& Colombini, 1993; Liu et al., 1994; Stobienia et al., 2002) but also with intact mitochondria (Liu \& Colombini, 1992; Stobienia et al., 2002). In the reconstituted systems, the VDAC modulator increases the voltage dependence of VDAC in three ways: it increases the rate of channel closure, decreases the rate of opening and induces the channels to assume lower conductance closed states. With intact mitochondria, the VDAC modulator was shown to partially inhibit transport of adenine nucleotides as well as NADH across the outer membrane when added to the cytoplasmic side. Thus, the VDAC modulator seems to mediate changes of VDAC conductance states also in native mitochondria. However, the responsible protein(s) has not been identified yet. Nevertheless, it has been shown recently that in the case of $S$. cerevisiae, depletion of VDAC does not eliminate the presence of the channel modulating activity in the intermembrane space although the presence of VDAC influences the properties of intermembrane space protein(s) (Stobienia et al., 2002).

Since the protein(s) responsible for VDACmodulating activity in the intermembrane space can act on the channel of native mitochondria also from the cytoplasmic side it is assumed that a similar modulating protein(s) might be present in the cytoplasmic compartment (Liu et al., 1994). Here we demonstrate that cytoplasm of both wild type and VDAC depleted ( $\Delta$ por 1 ) cells of $S$. cerevisiae indeed contains a protein fraction displaying VDAC-modulating activity (termed here cytoplasmic modulator fraction - CMF). In the reconstituted system CMF isolated from wild type as well as from $\Delta$ por 1 cells induces lower conductance levels of VDAC without statisti- 
cally significant differences. In the case of intact wild type mitochondria both CMF affect external NADH transport across the outer membrane in a way dependent on the mitochondria energized state although with quantitative differences which suggests that VDAC may influence the properties of cytoplasmic proteins.

\section{MATERIAL AND METHODS}

Yeast strains. A wild type strain of the yeast Saccharomyces cerevisiae M3 (MATa, lys2 his4 trp1 ade2 leu2 ura3) and VDAC1 (porin1)-depleted mutant M22-2 ( $\Delta$ por1) (Blachly-Dyson et al., 1997; Lee et al., 1998) were grown at $28^{\circ} \mathrm{C}$ in YPG medium ( $1 \%$ yeast extract, $2 \%$ peptone, $3 \%$ glycerol) at $\mathrm{pH} 5.5$.

Isolation of mitochondria and mitoplasts. Wild type mitochondria were isolated according to a published procedure (Daum et al., 1982). Mitoplasts were obtained by the swelling-shrinking procedure essentially as described in Daum et al. (1982). The estimation of the integrity of the outer membrane of wild type mitochondria was based on the permeability of the membrane to exogenous cytochrome $c$ (Douce et al., 1984) or on immunodecoration of Western blots with antisera against yeast marker proteins of the intermembrane space $\left(\mathrm{CC}_{1} \mathrm{HL}\right.$ - cytochrome $c_{1}$ heme lyase) and mitochondrial matrix (Mge1p). The calculated mean value of the degree of the outer membrane intactness was $96 \%$.

Isolation of VDAC-modulating activity-containing fraction (cytoplasmic modulator fraction - CMF). Proteins of the cytoplasmic compartment of wild type and $\Delta$ por 1 cells were fractionated by ammonium sulfate precipitation as described in Liu et al. (1994). After resuspension in the MDE buffer containing $10 \mathrm{mM}$ Mops/KOH pH 7.2, $1 \mathrm{mM}$ dithiothreitol (DTT) and $0.25 \mathrm{mM}$ EDTA, the collected fractions were tested in the reconstituted system (described below) for the pres- ence of a channel-forming activity and then for the presence of VDAC-modulating activity. To exclude contamination by mitochondrial proteins the active fraction devoid of any channel-forming activity (termed here cytoplasmic modulator fraction - CMF) was checked by Western blotting with anti-yeast antisera for the presence of marker proteins of mitochondrial compartments. They were as follows: Tom 40 and VDAC for the outer membrane, ADP/ATP carrier for the inner membrane, $\mathrm{CC}_{1} \mathrm{HL}$ for the intermembrane space and Mge1p for the mitochondrial matrix. The fraction was stored at $-80^{\circ} \mathrm{C}$.

Isolation of VDAC. The outer membrane released during mitoplast preparation was purified on a step $15-30-60 \%$ sucrose gradient buffered with $10 \mathrm{mM}$ Tris/ $\mathrm{HCl}, \mathrm{pH} 7.4$, and sedimented (Daum et al., 1982). The obtained outer membrane pellet was suspended in the solubilisation buffer containing $3 \%$ Triton $\mathrm{X}-100,10 \mathrm{mM}$ Tris/HCl (pH 7.0) and $1 \mathrm{mM}$ EGTA. The suspension was then loaded onto a dry hydroxyapatite/celite column and VDAC was eluted by the solubilisation buffer (De Pinto et al., 1987). Before reconstitution the preparations of VDAC were checked by Western blotting with anti-yeast antisera for the presence of ADP/ATP carrier (the inner membrane), Mge1p (the mitochondrial matrix) and $\mathrm{CC}_{1} \mathrm{HL}$ (the intermembrane space) as well as for VDAC and Tom40 (a major component of the TOM complex which displays a channel-forming activity).

Conductance measurements in planar phospholipid membranes. The planar phospholipid membrane experiments were performed according to (Benz et al., 1978). Membranes were formed from a $2 \%(\mathrm{w} / \mathrm{v})$ solution of soybean asolectin dissolved in $n$-decane, across a circular hole (surface area about $0.5 \mathrm{~mm}^{2}$ ) in the thin wall of a Teflon chamber separating two compartments (cis-trans) filled with unbuffered $1 \mathrm{M} \mathrm{KCl,} \mathrm{pH} \mathrm{7.0.} \mathrm{The} \mathrm{cham-}$ ber was connected with the recording equipment through calomel half-cells. All preparations described above were added in small 
aliquots (2-9 $\mu \mathrm{l})$ to the cis compartment. Cis also refers to the compartment where the voltage was held. The amplified signal was monitored with an oscilloscope and recorded on a strip chart recorder.

Determination of the rate of $N A D H$ oxidation. Mitochondria or mitoplasts were incubated in SM buffer containing $250 \mathrm{mM}$ sucrose, $20 \mathrm{mM} \mathrm{KCl}, 10 \mathrm{mM}$ Mops/KOH pH 7 . $2.5 \mathrm{mM} \mathrm{MgCl}_{2}, 3 \% \mathrm{BSA}$, in the presence of different external NADH concentrations and $135 \mu \mathrm{g}$ of CMF per $50 \mu \mathrm{g}$ of mitochondrial protein (Liu \& Colombini, 1992). To trigger the uncoupled state (state $U$ ), valinomycin at a concentration of $0.16 \mu \mathrm{g}$ per mg of mitochondrial protein was added to the incubation. NADH oxidation was monitored spectrophotometrically (UV 1602 Shimadzu).

Other methods. Protein concentration was measured by the method of Bradford. Respiration of mitochondria and mitoplasts was monitored at $25^{\circ} \mathrm{C}$ with a Rank oxygen electrode in the incubation volume of $0.5 \mathrm{ml}$. Changes of the inner membrane potential $(\Delta \psi)$ were monitored with a tetraphenylophosphonium $\left(\mathrm{TPP}^{+}\right)$- specific electrode as described in (Kamo et al., 1979). CMF isolated from wild type and $\Delta$ por 1 cells were subjected to SDS/PAGE (Laemmli, 1970) and the obtained gels were stained with Coomassie Brilliant Blue G250. Protein identification was performed by mass spectrometry (Mass Spectrometry Laboratory, Institute of Biochemistry and Biophysics PAS, Warsaw, Poland).

\section{RESULTS AND DISCUSSION}

Taking into account that cytoplasm might contain a VDAC-modulating activity similar to that present in the mitochondrial intermembrane space we fractionated proteins of the $S$. cerevisiae cytoplasmic compartment by the method applied in the case of the intermembrane space proteins (Liu et al., 1994; Stobienia et al., 2002). The fraction analogous to the intermembrane space pro- tein fraction displaying VDAC-modulating activity but not contaminated by mitochondrial proteins (as was checked by Western blotting - not shown) was termed the cytoplasmic modulator fraction (CMF). It was isolated from wild type as well as from VDAC depleted ( $\Delta$ por 1$)$ cells $(\mathrm{CMFwt}$ and CMF $\Delta$ por 1, respectively) and its influence on VDAC permeability was first analysed in a reconstituted system, i.e. on VDAC reconstituted in a black membrane bilayer made of asolectin.

It is well known that VDAC behavior in planar phospholipid membranes made of asolectin is symmetrical (Colombini, 1994; Colombini et al., 1996). This means that the channel closes at about the same rate and to about the same extent independently on the sign of the applied potential. On the other hand, the VDAC modulator isolated from the mitochondrial intermembrane space acts from both sides of VDAC incorporated into planar membranes provided the modulator-containing side is made negative (Elkeles et al., 1997; Holden \& Colombini, 1988; Liu \& Colombini, 1992; Holden \& Colombini, 1993; Liu et al., 1994). Therefore, in our experiments a membrane potential of $-10 \mathrm{mV}$ at the cis side was imposed (see Materials and Methods). Further, all measurements were made in the presence of a standard solution of $1 \mathrm{M} \mathrm{KCl}$.

Figure 1A shows the obtained distribution of conductances of the reconstituted VDAC (98 single insertions) in the absence of modulating fractions. Under the applied conditions two main values of conductances were obtained; namely $3.85 \pm 0.27$ (83\% of all insertions) and $2.21 \pm 0.33$ (17\% of all insertions). This denotes the dominance of a fully open state over multiple closed states (Benz, 1994) which is consistent with published data (Holden \& Colombini, 1988; Liu \& Colombini, 1992). Addition of CMFwt to the cis side, at a final protein concentration of approximately $30 \mu \mathrm{g} / \mathrm{ml}$, distinctly increased the lower VDAC conductances, i.e. the probability of VDAC closing. For 102 single insertions two 
main values of conductance were calculated, namely $4.21 \pm 0.31$ and $2.19 \pm 0.30$ with a ratio of $48 \%$ to $52 \%$, respectively. This corresponds to the dominance of closed states. Thus, the effect of CMFwt resembles that imposed by the VDAC modulator isolated from the mitochondrial intermembrane space (Stobienia et al., 2002), although is distinctly weaker. Moreover, the active cytoplasmic protein fraction has to be applied in higher amounts (10 times higher). This probably results from a wider diversity of cytoplasmic proteins and, as a consequence, lower content of the CMF in comparison with the intermembrane space proteins. Nevertheless, one might conclude that the cytoplasm of $S$. cerevisiae cells contains protein(s) able to modulate VDAC in a way similar to the VDAC modulator located in the intermembrane space of mitochondria.

We next investigated whether the expression of VDAC-modulating activity in the cytoplasm might depend on the presence of the channel in the mitochondrial outer mem- brane. For that purpose we analysed the influence of CMF $\triangle$ por 1 on the reconstituted VDAC. As shown in Fig. 1C, a histogram of 105 insertion events in the presence of CMF $\Delta$ por 1, added to the cis side at a final protein concentration of approximately 30 $\mu \mathrm{g} / \mathrm{ml}$, revealed two main values of conductance; namely $4.07 \pm 0.57$ (58\% of all insertions) and $2.14 \pm 0.28$ ( $42 \%$ of all insertions). These are comparable to those calculated for the reconstituted VDAC in the presence of CMFwt (Fig. 1B). Moreover, analysis of distributions of VDAC conductance values in the presence of CMFwt and CMF $\triangle$ por 1 by t-Test (at $\mathrm{t}=2,17117 \mathrm{p}=0,05267$ and $\alpha=0,01$ ) indicated that the obtained differences were not significantly different. Thus, under the applied conditions, no difference in CMFwt and CMF $\Delta$ por 1 action on the reconstituted VDAC permeability is observed. This means that the probability of VDAC transition into low conductance states is in principle the same in the presence of CMFwt and CMF $\Delta$ por 1. There-
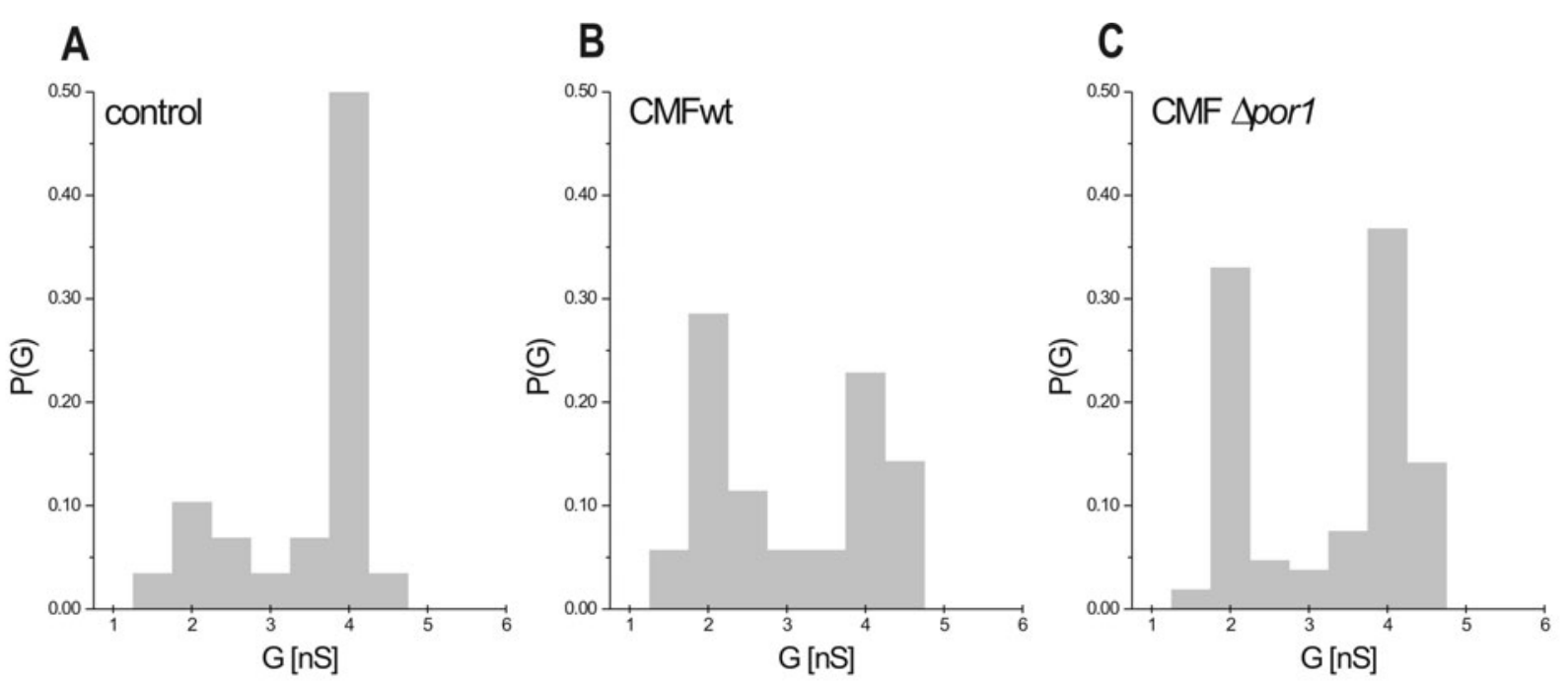

Figure 1. Histograms of VDAC conductances calculated in the presence of fractions containing VDAC-modulating activity isolated from the cytoplasm of wild type and $\Delta$ por 1 cells (CMFwt and CMF $\Delta$ por 1, respectively).

A: Control measurements in the absence of modulating fractions. B: VDAC conductances in the presence of CMFwt. $\mathrm{C}$ : VDAC conductances in the presence of CMF $\Delta$ por 1 . In all cases single channel conductances were measured at a membrane potential of $-10 \mathrm{mV}$. CMFwt or CMF $\Delta$ por 1 were added at a final protein concentration of approximately $30 \mu \mathrm{g} / \mathrm{ml}$. The data represent mean conductances of the following number of insertion events: 98 (A), 102 (B) and $105(\mathrm{C}) . \mathrm{P}(\mathrm{G})$ is the probability that a given conductance increment $\mathrm{G}$ is observed. 
fore, one might conclude that the expression of cytoplasmic protein(s) enhancing VDAC closure is independent of the presence of the channel in the mitochondrial outer membrane.

We have shown recently that the VDAC modulator isolated from the mitochondrial intermembrane space incubated with intact $S$. cerevisiae mitochondria reduces their capability to oxidise external NADH in a way dependent on the presence of the inner membrane potential $(\Delta \psi)$ (Stobienia et al., 2002). The oxidation of external NADH by $S$. cerevisiae mitochondria enables simple estimation of metabolite passage through the outer membrane (Lee et al., 1998; Michejda et al., 1994) since the substrate is oxidised by a dehydrogenase located on the outer surface of the inner membrane (De Vries \& Marres, 1987). Further, the main pathway for external NADH across the outer membrane of $S$. cerevisiae mitochondria is formed by VDAC (Lee et al., 1998). Thus, inhibition of external NADH oxidation by S. cerevisiae mitochondria reflects constraints imposed by the VDAC modulator isolated from the intermembrane space on VDAC permeability in intact mitochondria. On the other hand, the dependence of the observed effects on the presence of the inner membrane potential indicates that the voltage dependence of VDAC is not only confined to the reconstituted system. It is well known that $\Delta \psi$ may contribute to the existence of a potential across the outer membrane (Benz, 1994; Lemeshko, 2002) although changes in mitochondria morphology triggered by changes in $\Delta \psi$ might influence more directly the generation of the potential across the outer membrane and consequently the permeability of VDAC (Liu \& Colombini, 1991; 1992; Colombini, 1994). Therefore, to study the influence of CMF isolated from wild type and $\Delta$ por 1 cells on VDAC permeability in intact $S$. cerevisiae mitochondria we chose the oxidation of external NADH and performed experiments with coupled $(+\Delta \psi)$ and uncoupled $(-\Delta \psi)$ mitochondria. As we explained previ- ously (Stobienia et al., 2002), the term "coupled mitochondria $(+\Delta \psi)$ " denotes respiration induced by the addition of a respiratory substrate in the absence of ADP while the term "uncoupled mitochondria $(-\Delta \psi)$ " corresponds to respiration but in the presence of an uncoupler (valinomycin at a concentration of $0.16 \mu \mathrm{g}$ per $\mathrm{mg}$ of mitochondrial protein). Based on preliminary calibration experiments, performed for coupled wild type mitochondria in the presence of $140 \mu \mathrm{M}$ external $\mathrm{NADH}$, we chose CMF concentration of 135 $\mu \mathrm{g}$ per $50 \mu \mathrm{g}$ of mitochondrial protein. The concentration is approximately 30 times higher than that applied in the case of the VDAC modulator isolated from the mitochondrial intermembrane space but ensures comparable levels of inhibition of external NADH oxidation. This discrepancy results probably from the qualitative and quantitative dominance of cytoplasmic over intermembrane space proteins.

As shown in Fig. 2A, CMFwt inhibited external NADH oxidation by wild type mitochondria both in the presence and in the absence of $\Delta \psi$. Further, up to the external NADH concentration of approximately $70 \mu \mathrm{M}$, CMFwt imposed the same effect on the substrate oxidation by both coupled and uncoupled mitochondria, i.e. a fast increase in the inhibition up to approximately $40 \%$. At higher external $\mathrm{NADH}$ concentrations a further increase in the inhibition proceeded but at a much slower rate, particularly in the case of uncoupled mitochondria. Thus, in the presence of $560 \mu \mathrm{M}$ external NADH the levels of inhibition reached approximately $70 \%$ and $50 \%$ for coupled and uncoupled mitochondria, respectively. In the presence of CMF $\Delta$ por 1 (Fig. 2B), the same biphasic mode of inhibition of external NADH oxidation by wild type mitochondria was observed, i.e. a fast increase up to the substrate concentration of approximately 70 $\mu \mathrm{M}$ and then a second slower phase, independently of the energised state of mitochondria. The first phase was in principle identical for both coupled and uncoupled mitochondria 

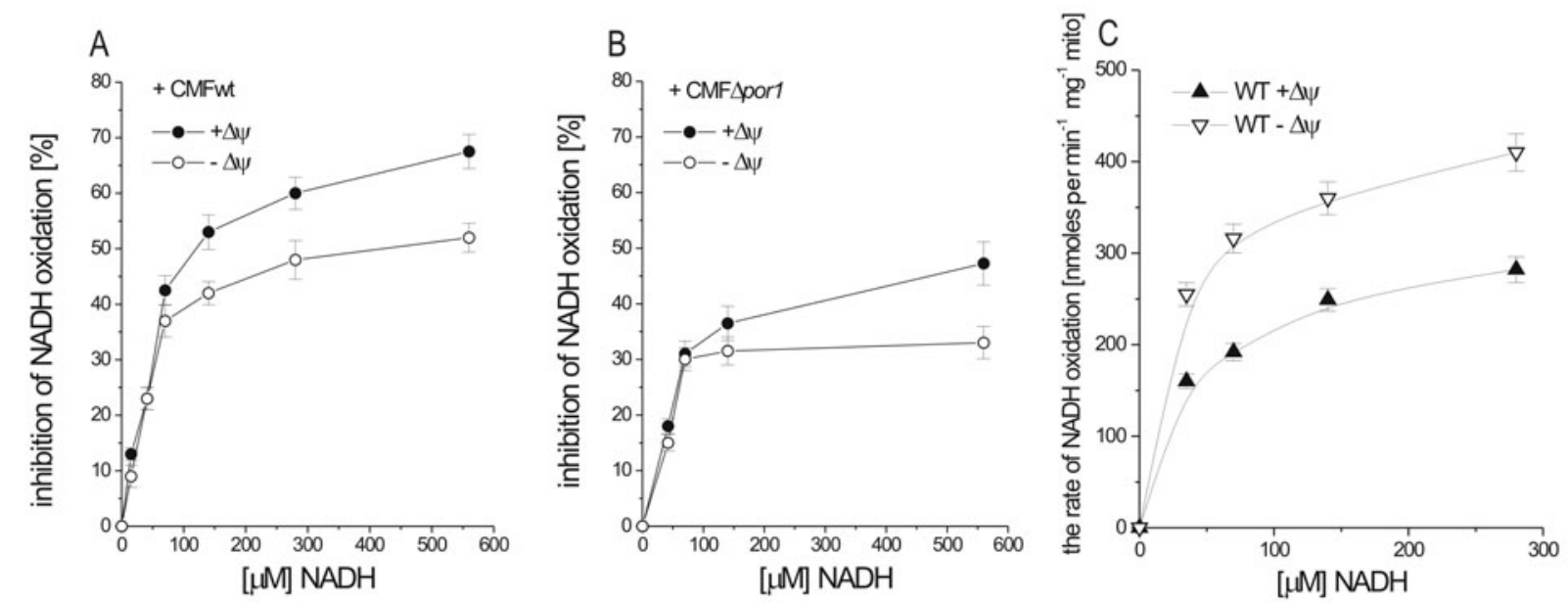

Figure 2. Inhibition of external NADH oxidation by wild type mitochondria imposed by fractions containing VDAC-modulating activity isolated from the cytoplasm of wild type and $\Delta$ por 1 cells (CMFwt and CMF $\triangle$ por 1, respectively).

Wild type mitochondria (50 $\mu \mathrm{g}$ of protein) were incubated in SM buffer in the presence of $135 \mu \mathrm{g}$ of a given CMF (or a proper volume of MDE buffer as the respective control) as described in Materials and Methods. To obtain uncoupled mitochondria valinomycin was used at a concentration of $0.16 \mu \mathrm{g}$ per $\mathrm{mg}$ of mitochondrial protein. Different concentrations of external NADH were applied and its oxidation was recorded spectrophotometrically in the presence or absence of the inner membrane potential, $+\Delta \psi$ or $-\Delta \psi$, respectively. A: Inhibition of external NADH oxidation in the presence of CMFwt. B: Inhibition of external NADH oxidation in the presence of CMF $\Delta$ por 1. C. The rates of external NADH oxidation calculated for coupled and uncoupled mitochondria incubated in the absence of CMF. Data are mean values \pm S.E.M. of ten independent experiments.

with the levels of inhibition of approximately $30 \%$ at $70 \mu \mathrm{M}$ external $\mathrm{NADH}$ while at higher concentrations of the substrate the inhibition was much less pronounced, especially in the case of uncoupled mitochondria. In the presence of $560 \mu \mathrm{M}$ external NADH the inhibition levels were approximately $45 \%$ and $35 \%$ for coupled and uncoupled mitochondria, respectively. Because lower rates of external NADH oxidation were observed for coupled mitochondria (Fig. 2C) one could conclude that the rate of the substrate transport through the outer membrane is not crucial to the action of both CMF. Thus, in the absence of $\Delta \psi$, the effects of CMFwt and CMF $\triangle$ por 1 are slightly weaker than in the presence of $\Delta \psi$, provided higher concentrations of external NADH are applied (above $70 \mu \mathrm{M}$ ). The results are distinctly different from that obtained in the presence of the VDAC modulator isolated from the intermembrane space. The modulator is totally inactive with uncoupled mitochondria, independently of whether it is isolated from wild type or $\Delta$ por 1 mitochondria (Stobienia et al., 2002). On the other hand, in the case of coupled mitochondria, the effect of both types of the modulator on external NADH oxidation also consists of two phases. These are a phase of fast inhibition and a slower phase of an increased inhibition (the modulator isolated from wild type mitochondria) or a decreased inhibition (the modulator isolated from $\Delta$ por1 mitochondria). Therefore, there are distinct qualitative differences between VDAC-modulating activities isolated from the mitochondrial intermembrane space and the cytoplasm. However, the quantitative differences in the modulating influence of 
CMFwt and CMFApor1 resemble those obtained for the VDAC modulator isolated from wild type and $\Delta$ por 1 mitochondria (Stobienia et al., 2002). The differences were not observed in the case of reconstituted VDAC and might be triggered by the presence of other mitochondrial protein(s) absent from the reconstituted system. Thus, a relation between the presence of VDAC and the properties of cytoplasmic proteins might exist.

To check whether the absence of VDAC in the outer mitochondrial membrane might influence the levels or diversity of cytoplasmic proteins we compared CMFwt and CMF $\Delta$ por 1 by SDS/PAGE. As shown in Fig. 3, the only visible difference consisted in upregulation of some proteins of approximate molecular mass from 35 to $50 \mathrm{kDa}$ observed in the case of CMF $\Delta$ por 1 . We managed to identify one of the proteins by mass spectrometry. It is 3-phosphoglycerate kinase responsible for one of the two ATP synthetic reactions in glycolysis (marked with an arrow). It is possible to speculate that an increased activity of 3-phosphoglycerate kinase in CMF $\Delta$ por 1 may prevent VDAC closing. The statement is in agreement with the weaker inhibition of external NADH oxidation by wild type mitochondria in the presence of the fraction (Fig. 2) but it is more probable that the effect of the enzyme is rather indirect. Undoubtedly further studies must be performed to identify the protein(s) responsible for VDAC-modulating activity located in the cytoplasm and the research is under way. On the other hand, the upregulation seems to be a general strategy applied by $S$. cerevisiae cells depleted of VDAC as also some mitochondrial proteins are upregulated in $\Delta$ por 1 cells (Kmita \& Budzińska; 2000; Antos et al., 2001).

Summing up, the obtained results hint at the existence of a VDAC-modulating activity in the cytoplasm of $S$. cerevisiae cells which seems different from the VDAC modulator located in the mitochondrial intermembrane space. It is likely that the proteins modulating VDAC from both its sides may together consti- tute an efficient machinery regulating the mitochondrial outer membrane permeability for metabolites. Our preliminary results (not shown) indicate that the studied cytoplasmic protein fractions might also be able to modu-

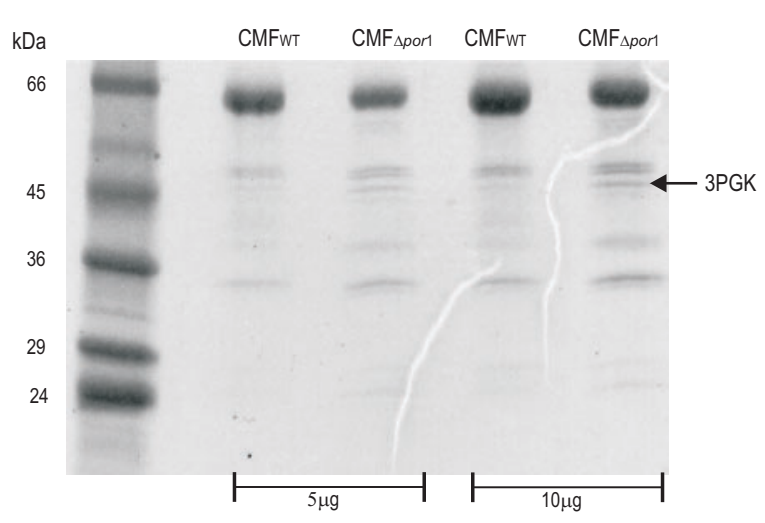

Figure 3. SDS/polyacrylamide gel electrophoresis of fractions containing VDAC-modulating activity isolated from the cytoplasm of wild type or $\Delta$ por 1 cells (CMFwt and CMF $\triangle$ por 1, respectively).

SDS/PAGE was performed in $12 \%$ gels. Proteins were visualized by staining with Coomassie Brilliant Blue G250. The figure presents typical results obtained with about 5 and $10 \mu \mathrm{g}$ of the studied fractions.

late the TOM complex channel (channel of the translocase of the outer membrane) which participates in protein translocation (our preliminary results). Therefore, identification of the proteins is very important.

The authors wish to thank Prof. M. Forte for the yeast strains. The technical assistance of D. Drachal-Chrul is gratefully acknowledged.

\section{R E F E R E N C E S}

Antos N, Budzinska M, Kmita, H. (2001) An interplay between the TOM complex and porin isoforms in the yeast Saccharomyces cerevisiae mitochondria. FEBS Lett.; 500: 12-6.

Benz R. (1994) Permeation of hydrophilic solutes through mitochondrial outer membranes: review on mitochondrial porins. Biochim Biophys Acta.; 1197: 167-96. 
Benz R, Janko K, Boos W, Lauger P. (1978) Formation of large, ion-permeable membrane channels by the matrix protein (porin) of Escherichia coli. Biochim Biophys Acta.; 511: 305-19.

Blachy-Dyson E, Forte M. (2001) VDAC channels. IUBMB Life.; 52:113-8.

Blachly-Dyson E, Song J, Wolfgang WJ, Colombini M, Forte M. (1997) Multicopy suppressors of phenotype resulting from the absence of yeast VDAC encode a VDAC-like protein. Mol Cell Biol.; 17: 5727-38.

Blachly-Dyson E, Zambrowicz EB, Yu WH, Adams V, McCabe ERB, Adelman J, Colombini M, Forte M. (1993) Cloning and functional expression in yeast of two human isoforms of the outer mitochondrial membrane channel, the voltage-dependent anion channel. J Biol Chem.; 268: 1835-41.

Colombini M. (1979) A candidate for the permeability pathway of the outer mitochondrial membrane. Nature.; 279: 643-5.

Colombini M. (1994) Anion channels in the mitochondrial outer membrane. Curr Top Membr.; 42: 73-101.

Colombini M, Blachly-Dyson E, Forte, M. (1996) VDAC, a channel in the outer mitochondrial membrane. In Ion Channels. Narahashi T. ed, pp 169-201. Plenum Press, New York.

Crompton M, Barksby E, Johnson N, Capano M. (2002) Mitochondrial intermembrane junctional complexes and their involvement in cell death. Biochimie; 84: 143-52.

Daum G, Bohni PC, Schatz G. (1982). Import of proteins into mitochondria. Cytochrome b2 and cytochrome c peroxidase are located in the intermembrane space of yeast mitochondria. J Biol Chem.; 257: 13028-33.

De Pinto V, Ludwig O, Krause J, Benz R, Palmieri F. (1987) Porin pores of mitochondrial outer membranes from high and low eukaryotic cells: biochemical and biophysical characterization. Biochim Biophys Acta.; 894: 109-19.

De Vries S, Marres CA. (1987) The mitochondrial respiratory chain of yeast. Structure and biosynthesis and the role in cellular me- tabolism. Biochim Biophys Acta.; 895: 205-39.

Douce R, Bourguignon R, Neuberger M. (1984) Isolation of plant mitochondria. General principles and criteria of integrity. Methods Enzymol.; 148: 403-15.

Elkeles A, Breiman A, Zizi M. (1997) Functional differences among wheat voltage-dependent anion channel (VDAC) isoforms expressed in yeast. Indication for the presence of a novel VDAC-modulating protein? J Biol Chem.; 272: 6252-60.

Heins L, Mentzel H, Schmid A, Benz R, Schmitz UK. (1994) Biochemical, molecular, and functional characterization of porin isoforms from potato mitochondria. J Biol Chem.; 269: 26402-10.

Holden MJ, Colombini M. (1988) The mitochondrial outer membrane channel, VDAC, is modulated by a soluble protein. FEBS Lett.; 241: 105-9.

Holden MJ, Colombini M. (1993) The outer mitochondrial membrane channel, VDAC, is modulated by a protein localized in the intermembrane space. Biochim Biophys Acta.; 1144: 396-402.

Kamo N, Muratsugu M, Hongoh R, Kobatake YJ (1979) Membrane potential of mitochondria measured with electrode sensitive to tetraphenyl phosphonium and relationship between proton electrochemical potential and phosphorylation potential in steady state. $J$ Membr Biol.; 49: 105-21.

Kmita H, Budzińska M. (2000) Involvement of the TOM complex in external NADH transport into yeast mitochondria depleted of mitochondrial porin1. Biochim Biophys Acta.; 1509: 86-94.

Laemmli UK. (1970) Cleavage of structural proteins during the assembly of the head of bacteriophage T4. Nature.; 227: 680-5.

Lee AC, Xu X, Blachly-Dyson E, Forte M, Colombini M. (1998) The role of yeast VDAC genes on the permeability of the mitochondrial outer membrane. J Membr Biol.; 161: 173-81. 
Lemeshko VV. (2002) Model of the outer membrane potential generation by the inner membrane of mitochondria. Biophys J.; 82: 684-92.

Liu MY, Colombini M. (1991) Voltage gating of the mitochondrial outer membrane channel VDAC is regulated by a very conserved protein. Am J Physiol.; 260: C371-4.

Liu MY, Colombini M. (1992) Regulation of mitochondrial respiration by controlling the permeability of the outer membrane through the mitochondrial channel, VDAC. Biochim Biophys Acta.; 1098: 255-60.

Liu MY, Torgrimson A, Colombini M. (1994) Characterization and partial purification of the VDAC-channel-modulating protein from calf liver mitochondria. Biochim Biophys Acta.; 1185: 203-12.

Michejda J, Kmita H, Stobienia O, Budzińska M, Lauquin GJ-M. (1994) Restriction of metabolite permeation through the outer mitochondrial membrane of porin-deficient yeast mutant. In: molecular biology of mitochondrial transport system. Forte M, Colombini M. eds, pp 341-56. Springer-Verlag, Heidelberg.

Sampson MJ, Lovell RS, Craigen WJ. (1997) The murine voltage-dependent anion channel gene family. Conserved structure and function. J Biol Chem.; 272: 18966-73.

Schein SJ, Colombini M, Finkelstein A. (1976) Reconstitution in planar lipid bilayers of a voltage-dependent anion-selective channel. $J$ Membr Biol.; 30: 99-120.
Shimizu S, Ide T, Yanagida T, Tsujimoto Y. (2000) Electrophysiological study of a novel large pore formed by Bax and the voltage-dependent anion channel that is permeable to cytochrome $c$. J Biol Chem.; 275: 12321-5.

Stobienia O, Wróblewska S, Antos N, Budzińska M, Kmita H. (2002) The key role of the energized state of Saccharomyces cerevisiae mitochondria in modulations of the outer membrane channels by the intermembrane space proteins. J Bioenerg Biomembr.; 34: 507-16.

Tsujimoto Y, Shimizu S. (2002) The voltage-dependent anion channel: an essential player in apoptosis. Biochimie.; 84: 187-93.

Vander Heiden MG, Chandel NS, Li XX, Schumacker PT, Colombini M, Thompson CB. (2000) Outer mitochondrial membrane permeability can regulate coupled respiration and cell survival. Proc Natl Acad Sci U S A.; 97: 4666-71.

Vander Heiden MG, Chandel NS, Schumacker PT, Thompson CB (1999) Bcl-xL prevents cell death following growth factor withdrawal by facilitating mitochondrial ATP/ADP exchange. Mol Cell.; 3: 159-67.

Xu X, Decker W, Sampson MJ, Craigen WJ, Colombini M. (1999) Mouse VDAC isoforms expressed in yeast: channel properties and their roles in mitochondrial outer membrane permeability. J Membr Biol.; 170: 89-102. 